\title{
Critical Reflections on Mental Well-being for Post-Secondary Students Participating in the Field of Global Health
}

\author{
Corey McAuliffe ${ }^{1}$ (D) Ross Upshur ${ }^{2} \cdot$ Daniel W. Sellen $^{1} \cdot$ Erica Di Ruggiero ${ }^{1,3}$ \\ Published online: 23 October 2018 \\ (C) Springer Science+Business Media, LLC, part of Springer Nature 2018
}

\begin{abstract}
The ways in which global health students experience trauma/distress while conducting global health fieldwork is understudied. No identifiable literature addresses the risks to students' mental well-being, although physical wellness checks exist. Importantly, global health practitioners are at greater risk than the general population for moral distress, secondary-traumatic stress disorder, vicarious traumatization, compassion fatigue, burnout, stress, and anxiety. Students face increased risks (e.g., illness, physical safety), especially in respect to their mental well-being. While academic institutions often require pre-departure trainings, research suggests that they are insufficient or ineffective. Challenges are not only limited to before and during one's placement, but can be intensified after returning home due to concerns about one's reputation, being perceived as not able to cope, or feeling ethically/morally obliged to continue research that can be re-traumatizing. This paper critically reflects on these knowledge gaps and on how the lack of appropriate resources and supports has ethical and practical implications for students, the wider academic community, and the communities in which they work.
\end{abstract}

Keywords Global health fieldwork $\cdot$ Post-secondary students $\cdot$ Mental health $\cdot$ Distress $\cdot$ Trauma Academic institutions

Corey McAuliffe

corey.mcauliffe@mail.utoronto.ca

1 Division of Social and Behavioural Health Sciences, Dalla Lana School of Public Health, University of Toronto, 155 College Street, Toronto, ON M5T3M7, Canada

2 Division of Clinical Public Health, Dalla Lana School of Public Health, University of Toronto, Toronto, ON, Canada

3 Office of Global Public Health Education \& Training, University of Toronto, Toronto, ON, Canada 


\section{Field of Global Health}

Content warning: this article includes content pertaining to traumatic and distressing experiences of individuals, including sexual violence. In recent decades, global health has undergone dramatic growth within academic institutions, expanding education programs and partnerships between institutions in high-income countries with low- and middle-income countries; through the development of global health competencies; and incorporation of internationally-based service, practicum, and research opportunities (Brown et al. 2006; Di Ruggiero et al. 2018; Macfarlane et al. 2008; Matheson et al. 2014; Pinto and Upshur 2013). According to Koplan et al.'s (2009) frequently cited definition, "global health is an area for study, research, and practice that places a priority on improving health and achieving equity in health for all people worldwide" (p. 1995). These global health activities most often originate within schools of public health and medicine; however, other disciplines are increasingly participating in these endeavors, "including nursing, anthropology, engineering, political science, sociology, theology, and veterinary medicine" (Matheson et al. 2014, p. 4).

As academic programs continue to receive an influx of student applications, the desire for access to internationally-based global health experiences and placements also continues to grow. This, in part, is due to student demand at all levels of post-secondary education (undergraduate to graduate to medical residency), and will likely continue to grow, as the number of institutions offering an undergraduate global health major has been estimated to more than double between 2014 and 2019 (McKinzie 2017). With a growing number of students participating in global health, access to applied practical experiences are being sought after, at unprecedented levels. These opportunities are often invaluable to training and essential to furthering one's career. Global health leaders and faculty also promote global health opportunities as effective recruitment and retention tools (McKinzie 2017). While the benefits of internationally-based global health placements are often discussed, this critical review synthesizes the available literature, highlighting that these experiences do not come without significant mental, emotional, and physical health risks.

\section{Post-Secondary Mental Health}

As previously documented, mental and emotional well-being is a growing concern on North American university campuses (Mountz et al. 2015; National Council on Disability 2018; Parizeau et al. 2016; Peake and Mullings 2016). Surveys indicate that all post-secondary students and faculty are at an elevated risk for anxiety, depression, stress, feelings of isolation, and suicidal ideation (Mountz et al. 2015; Parizeau et al. 2016). Structural violence and oppression due to sex, gender, class, abilities, and sexual orientation can produce or exacerbate experiences of trauma and distress, and lead to increased challenges to mental well-being (McKinzie 2017). For students, these challenges have been linked, in part, to unsustainable demands and unreasonable expectations driven by neoliberal universities, such as the rising cost of education, expectations of high/increased productivity, and pressures to finish within compressed periods of time (Mountz et al. 2015). According to the 2018 National Council on Disability's Mental Health Report, universities are struggling "to provide adequate mental health services and supports for students with mental health disabilities due largely" to increased numbers of mental health reports by students and constrained institutional financial means (National Council on Disability 2018). Students are often placed on 
waiting lists due to insufficient staffing and increased need for services when attempting to access university mental health services (National Council on Disability 2018).

Comparatively, these experiences are often intensified for graduate students, due to increased social isolation, feelings of guilt, and imposter syndrome (feelings of inadequacy or chronic selfdoubt despite external proof of success), financial concerns, sexual harassment and abuse by advisors and colleagues, competition and pressure to develop CVs, and future job insecurity (McKinzie 2017; National Council on Disability 2018; Peake and Mullings 2016). When distress occurs, "it can be debilitating and can lead to depression, fatigue, depersonalization, and burnout" (Surya et al. 2017, p. 189). Even more troubling are recent research findings, pointing to the development of a psychiatric condition in more than $30 \%$ of $\mathrm{PhD}$ students (Levecque et al. 2017). While mental health is a major concern for all academics, there has been little consideration to the additional negative health impacts to students working in the field of global health. The implications and consequences of students' experiences of stress, anxiety, panic, and trauma due to internationallybased practicums are rarely discussed and need significant attention. The perspectives documented in this paper largely reflect the experiences of students and faculty based in high-income country institutions rather than those of students from low- and middle-income countries.

\section{Risks}

\section{Internationally-Based Global Health Practice}

Many placements within the field of global health take place in low- and middle-income countries. Training opportunities are often located in unfamiliar and precarious environments, where students regularly undertake unpaid or underpaid work, with limited support and resources. Within these placements, there are inherent risks such as access to appropriate healthcare, language barriers, increased risk of illness (e.g., foodborne, infectious disease), physical safety (e.g., car accidents, theft, violence), and mental health conditions (e.g., anxiety, depression, culture shock) (Calgaro 2015; Dominey-Howes 2015; Drozdzewski and Dominey-Howes 2015; Rowley et al. 2007). Additionally, in interviews with university global health leaders, participants acknowledged that there are "potential risks to students and faculty engaged in overseas activities, where threats of violence, illness, or accidents may be higher than at home" (Matheson et al. 2014, p. 13). However, while culture shock, transport accidents, and tropical illnesses are commonly and implicitly understood as standard fieldwork risks, they can also be experienced as challenging, distressing, or traumatic (Paulson et al. 2009; Pollard 2009). Furthermore, there are additional hardships faced by female students (e.g., sexual violence, harassment, gender-based discrimination, (in)fertility). These challenges may be intensified for all students after returning home, due to concerns about one's reputation, being seen as not able to cope, or feeling ethically or morally obliged to continue research that is retraumatizing (e.g., transcription, analysis, writing up phase) (Paulson et al. 2009).

Within the literature, there are no identifiable institutional sources that systematically address researchers' mental well-being while working in global health, although physical wellness checks do exist (e.g., vaccinations, physical wellness exams). There is limited research on the experience of students participating in global health in general, and few articles reflect on the vulnerability of women participating in this field (Hopwood and Paulson 2012; Paulson et al. 2009; Pollard 2009; Serekoane et al. 2014). Students represent a vulnerable and unique sub-population due to a lack of legal and social protections, afforded to those with 
comparable full time employment by North American organizations (including tenured faculty and staff). As such, there remains a lack of critical scholarship on understanding student experiences and the resulting health and social impacts of global health practice.

\section{Humanitarian Aid and Relief Workers}

Students who participate in global health research may perform contextually similar work to humanitarian aid or relief workers, for instance, assisting refugees, working with traumatized populations, or victims of violence, war, and sexual assault. Much can therefore be gleaned from the literature on their experiences. As a whole, global health practitioners are at greater risk than the general population for moral distress (Huffman and Rittenmeyer 2012; Klocker 2015), depression (Surya et al. 2017), secondarytraumatic stress disorder, vicarious traumatization (Cohen and Collens 2013; Jenkins and Baird 2002), post-traumatic stress disorder (PTSD), compassion fatigue, burnout, stress, and anxiety (Breslau and Kessler 2001; Connorton et al. 2012; Rowley et al. 2007). Specifically, mental well-being is imperative for global health practitioners' "ability to function effectively, particularly when exposed to extreme environments" (Surya et al. 2017, p. 188) as "emergencies erode normally protective supports, increase the risk of diverse problems and tend to amplify preexisting problems of social injustice and inequality" (Inter-Agency Standing Committee 2006 as cited in Surya et al. 2017, p. 191). According to the 2013 United Nations High Commissioner for Refugees's (UNHCR) Mental Health and Psychosocial Support for Staff, 89\% of survey respondents reported that they have currently or within the last month experienced some type of distress. According to these online respondents, who were currently on a field mission in Bangladesh and Pakistan, around one in every two respondents reported feelings of sadness, unhappiness, or "emptiness"; irritability or frustration, even over small matters; fatigue, tiredness, and loss of energy; and difficulty sleeping or sleeping more than usual (Welton-Mitchell 2013, p. 56).

Within many humanitarian aid and relief worker studies, authors identified that most agencies did not provide pre-service mental health screenings, social or psychological support during fieldwork, or post-service care based on varied reasons (e.g., expectation of worker's adaptability, limited resources, aid worker seen as an expendable asset, less important for aid workers than that of the target population) (Cardozo et al. 2005; Connorton et al. 2012; Ditzler 2001; Macfarlane et al. 2008). According to Surya et al. (2017), as complex global health crises arise, "local and expatriate health professionals have become increasingly exposed to stress and trauma for protracted periods" (p. 188). Studies have indicated that "health professionals working in extreme settings are vulnerable to symptoms of PTSD as a reaction to assisting traumatized and distressed individuals" (Surya et al. 2017, p. 189) and "that chronic high levels of stress and distress contribute to unhealthy coping methods, resulting in poor health and decreased overall efficiency" (Surya et al. 2017, p. 193). Data is also lacking on the number of international humanitarian aid workers who leave the global health field following repeated exposure to traumatic experiences. As the literature shows, there is a need to better prepare aid workers for coping with trauma and stress in the field; to provide adequate psychological support before, during, and after service; and to conduct rigorous research on the mental health outcomes related to trauma (Bauer and Thomas 2009; Connorton et al. 2012). 


\section{Contributing Factors: Before Fieldwork}

\section{Preparedness}

Travel and safety abroad offices are common in large academic institutions that deal with the practical implications of students working internationally. These offices often require students to participate in pre-departure trainings, and to carry emergency and disaster evacuation insurance. However, when offered, these trainings appear to more heavily weigh issues of physical well-being, ignoring or minimizing the potential mental and emotional impacts of this work. Regardless of their content, trainings are often seen as insufficient or ineffective, focus predominantly on theory rather than practice, lacking suggestions for adequate coping strategies, and leave students confused in terms of who to contact for support (Paulson et al. 2009; Pollard 2009; Serekoane et al. 2014). Furthermore, throughout their global health work, students overwhelmingly indicated that they feel isolated and ill-prepared, lacking adequate supervisory and organizational supports (Calgaro 2015; Donahoe et al. 2017; Pollard 2009). While these experiences may be exacerbated for students participating in solo research for the first time, Serekoane et al. (2014) noted that those with previous experiences, also often felt unprepared to deal with unanticipated challenges.

Academic researchers have pointed to a lack of attention on researcher protection. While ethical review boards exist to protect research participants, there are no similar boards and a dearth of institutional policies that consider the ethics and well-being of researchers and research trainees (Calgaro 2015; Dominey-Howes 2015). However, it must be noted that these issues are not limited to the time before and during one's fieldwork; rather, these challenges are often exacerbated by the realization that there is often little to no institutional support once researchers return from fieldwork placements (Pollard 2009; Serekoane et al. 2014). While debriefing rarely occurred, those who could share their distressing and challenging experiences with an empathetic peer, found the process to be cathartic (Calgaro 2015; Lowery and Stokes 2005). Within North American academic institutions, there are no universally applied institutional policies that predicate best or promising practices to support the physical, emotional, and mental health of students and faculty engaged in global health fieldwork. The lack of appropriate resources and support before, during, and after global health fieldwork has profound ethical and practical implications on students, the communities in which they work, and the broader academic community.

\section{Contributing Factors: During Fieldwork}

\section{Illness}

A few studies have documented the relationship between global health fieldwork and illness. In a recent longitudinal study on summer fieldwork, almost half of all global health students from a US Master of Public Health program reported at least one or more health problems, "with several students each year reporting serious events requiring hospitalization or evacuation from the host country" (Donahoe et al. 2017, p. 473). In other studies, UK and US graduate students reported stress headaches and migraines, fatigue, exacerbation of autoimmune conditions, contracting tropical diseases (typhoid, malaria, chikungunya, dengue fever, 
etc.), stomach issues (parasites, worms), and body pain (Begley 2009; Pollard 2009; Sohn 2017). As physical illness is a common risk in global health work, it is common for students to seek treatment if healthcare is accessible and affordable. However, when students are alone in an unfamiliar environment, they sometimes find it difficult to locate or access appropriate treatment, as well as to find effective support (Pollard 2009, p. 20). Furthermore, distressing and traumatizing experiences of ill health often leave invisible scars and have led to PTSD for some (Begley 2009; Sohn 2017).

\section{Emotional Challenges}

For those working on research that incorporates sensitive and emotional topics, there can be a "personal cost to researchers" (Dickson-Swift et al. 2007, p. 336), as this type of research can illicit deep personal reflection and acknowledgement of one's own vulnerability. Researchers may consciously or unconsciously hold on to participants' emotions and feelings, with little understanding of how to process and let go of them (Connolly and Reilly 2007). For instance, culturally appropriate expressions of emotion or cross-cultural understandings proved challenging to Calgaro (2015), a PhD geography student, who was unable to voice or express emotions while in Thailand, for fear that it would be "culturally-inappropriate and [seen as] a source of weakness by [her] Thai colleagues." This furthered Calgaro's experience of isolation, and propelled her to disengage from further social interactions.

\section{Mental Health Challenges}

For those dealing with mental and emotional challenges while abroad, confidentiality and disclosure prove particularly challenging (Tucker and Horton 2018). A sense of shame, as well as a need to "pass" or "cope" was often reported during this type of work (Pollard 2009; Tucker and Horton 2018). Daily stresses of cross-cultural misunderstandings or faux pas can be exacerbated by feelings of stress and anxiety due to heightened vigilance, insomnia, lack of nutrition, injury, and unknown illnesses (Calgaro 2015; Paulson et al. 2009; Pollard 2009; Sohn 2017). In a collection of qualitative studies, UK anthropology and social science doctoral students reported the following experiences while abroad, "anxiety, depression, high levels of stress, isolation, sleeplessness, sadness, and feelings of inadequacy," (Paulson et al. 2009, p. 2 ), as well as months of panic and fear, frustration, complete breakdowns, isolation, and loneliness (Begley 2009; Pollard 2009). Pollard (2009) further identified the following array of emotions reported by $\mathrm{PhD}$ anthropology students while working abroad: "alone, ashamed, bereaved, betrayed, depressed, desperate, disappointed, disturbed, embarrassed, fearful, frustrated, guilty, harassed, homeless, paranoid, regretful, silenced, stressed, trapped, uncomfortable, unprepared, unsupported, and unwell" (p. 1). These experiences ranged from temporary to long term, with only some students seeking out treatment.

Many students do not seek out treatment and counseling for a variety of reasons. Eriksen and Ditrich (2015) point out that those suffering from a "lower intensity at which vicarious traumatization can occur (compared with direct trauma) means many do not realise they are being affected." This trauma may be further exacerbated, as many global health practitioners report feeling isolated or disconnected from faculty, friends, and family (Pollard 2009). Other students noted a tacit expectation of "gritting one's teeth" or "putting on a brave face," as hardship and struggle was a perceived expectation to prove one's self-worth (Pollard 2009; Tucker and Horton 2018). This sentiment is apparent in 
Delamont's (2009) response to Pollard's (2009) study on the fieldwork experiences of $\mathrm{PhD}$ anthropology students, "the habitus of the discipline of anthropology relies on a widespread agreement that not everyone can be an anthropologist, and the survival of the misery and bafflement of fieldwork is the best way to see who is, and is not fit to join the culture" (p. 1). The inability to express intense emotions, not only has detrimental effects on the individual, but also on their daily practice, research and interaction with peers and community members (Calgaro 2015; Dominey-Howes 2015; Drozdzewski and DomineyHowes 2015).

\section{Sexual Violence}

The experience of sexual harassment, sexual violence, and gender-based discrimination plays a vital role in the mental well-being of global health practitioners. Whether explicitly discussed or addressed, gender dynamics are prevalent within all global health fieldwork (Sharp and Kremer 2006). Sexual harassment is a common experience within international placements, yet little has been done to address it (Hanson and Richards 2017; Kloß 2016). Female students reported that their physical safety was of particular concern, as some expressed feeling "very visible, and were vulnerable in public no matter how much they covered up their bodies" (Pollard 2009, p. 9). According to the Women in Global Health Research Initiative (Center for Global Health n.d.), "twenty-six percent of women report having experienced unwanted physical contact while doing international field research". In Clancy et al.'s (2014) Survey of Academic Field Experiences (SAFE): Trainees Report Harassment and Assault, 64\% of female respondents experienced sexual harassment, while $20 \%$ were victims of sexual assault. Those most likely to have experienced these forms of harassment and assault were female trainees, while perpetrators were most often senior male research team members (Clancy et al. 2014). Females expend precious energy and attention attempting to actively mitigate unwanted sexual advances and attention by dressing conservatively or in baggy, ill-fitting clothes; wearing a ring on one's wedding finger; avoiding personal relationships; living with a local family; and constantly monitoring one's own behavior (Congdon 2015; Grieser 2014; Johansson 2015; Kloß 2016; Ross 2015; Schwedler 2006; Sharp and Kremer 2006). However, these coping strategies are not always effective.

\section{Family Planning}

An additional strain for some female students, throughout their academic endeavors, is the decision of whether or not to have children during post-graduate education. The stress that comes along with this complex life event includes managing (in)fertility, child-bearing, and parenting and is affected by "gender, age, and career stage" (Parizeau et al. 2016, p. 10). These concerns and challenges can be further exacerbated when working in countries with increased rates of infectious disease that are specifically harmful before or during pregnancy (e.g., Zika, malaria). This may also involve pressures to disclose sensitive information to supervisors or other academic administrators that one may otherwise not want to divulge. For those with families, time spent away for global health travel increases the likelihood that female scientists will leave their careers at a higher rate than their male counterparts, due to child rearing responsibilities and other issues of work/life/family balance (Center for Global Health n.d.). Those who are single and/or childless and still of childbearing age, often face their own critics. Some communities who are used to women marrying and having children early in life, may be wary of lone, single female researchers, sometimes making it difficult for them to create supportive and trusting networks (Grieser 2014). 


\section{Contributing Factors: After Fieldwork}

\section{Mental and Emotional Challenges}

As documented above, it is evident that global health students are at high-risk for mental, physical, and emotional health conditions during their international work. However, what is less often discussed is the ongoing mental and emotional risks that students face once they return home. Beyond the fatigue, jet lag, and lack of recovery time from their experience (Tucker and Horton 2018), students struggle with merging a new sense of self, reverse culture shock, and returning to difficult data (and thus memories) (Begley 2009; Dominey-Howes 2015; Pollard 2009). The return home is often the first opportunity students have to critically reflect on their experience, often with mixed feelings. Frequently, there is little to no time to process what has happened or how one feels about it when they are in the midst of living it. For those who have become accustomed to a different way and pace of life, what is known as "reverse" culture shock, is sometimes experienced more jarringly than their work abroad (Pollard 2009). While reuniting with family and friends, there can still be feelings of isolation or an inability to convey life changing experiences to those who have not had similar experiences (Begley 2009).

\section{Returning to the Data}

One of the difficulties for graduate students returning home is having to return to their data, both analyzing and writing up findings, as part of degree requirements (Begley 2009; Pollard 2009). The experience of transcribing one's data can feel re-traumatizing or distressing. Students may elect to have outside transcriptionists involved in their academic research, who are also at elevated risk for secondary traumatic stress due to the "emotional impact that listening to data may cause" (Kiyimba and O'Reilly 2015, p. 93). As transcriptionists may not be an intimate part of the research team, they may have even more limited access to appropriate trainings, supports, or opportunities to debrief, even though their job demands that they listen to data repetitively and at length (Kiyimba and O'Reilly 2015). Global health fieldwork is often supported by a myriad of team members that are directly and indirectly connected to the collection and analysis of data. The potential negative health consequences of data collection and analysis to team members (e.g., community health workers, translators, transcriptionists, organizational staff, drivers) bring about critical ethical considerations.

For students, Pollard (2009) found that their process of writing-up was either cathartic or highly challenging, as reliving distressing experiences led to guilt, stress, and falling behind on timelines. Some people who participate in global health fieldwork experienced an altered perception or orientation of the world. For those who experienced a (dis)orientation, there is a need to address emotional responses that " 'loop back over and over' consciously and unconsciously shifting our positionality and changing how we approach our research" and interpret our findings (Drozdzewski and Dominey-Howes 2015). Additionally, considerations need to be given to researchers' personal well-being, especially when their research pertains to sensitive and emotionally charged data. Not only does ignoring emotions undermine the individual and their well-being, but it also greatly impacts the data collected, analyzed, and distributed (Drozdzewski and Dominey-Howes 2015). Yet, beyond the need to complete one's dissertation or subsequent manuscripts, positivistic instruction has created 
a world in which most academic literature is devoid of emotion. As England (1994) noted, "years of positivist-inspired training have taught us that impersonal, neutral detachment is an important criterion for good research" (Dominey-Howes 2015, p. 60). However, critical reflexivity in research needs to go beyond self-reflection and journal entries. Academics need to incorporate their emotions, traumas, distress, and embodied self within the process of research. This process can lead to new self-discovery, hypotheses, insights, and research questions, while helping researchers locate themselves within their fundamental assumptions (Dominey-Howes 2015).

\section{The Experience within Academia}

\section{Supervision}

Supervisors, committee members, and faculty mentors play an important role in the success and well-being of their students and supervisees. Paulson et al. (2009) found that supervisorial relationships were critical to students' well-being, and "that students experienced much distress when that relationship failed or was tense" (p. 3). Some UK doctoral students participating in global health research reported having little to no contact with their supervisors or committees while in the field, in addition to having limited contact with family and friends due to geographical remoteness (Paulson et al. 2009; Pollard 2009). Others felt silenced by their supervisors, and pressured to show how difficulties had helped them to grow professionally. For others still, supervisors provided support and a much-needed grounding for students (Pollard 2009).

While some awareness and attention have been brought to the needs of post-secondary students' mental well-being, the same has not been true for faculty members (Pettit 2016). In Dominey-Howes' (2015) experience as a supervisor, he identified the concept of "indirect professional" vicarious trauma, which occurred due to his supervision of $\mathrm{PhD}$ geography students who encountered difficult, distressing, and sometimes traumatizing experiences through their research interviews. While many supervisors may have had their own daunting experiences as $\mathrm{PhD}$ students, many have little to no training or support in how to deal with trauma or distress (their own or someone else's). Dominey-Howes' (2015) and Parizeau et al. (2016) further identified a lack of training, resources, and tools necessary to provide better support to their supervisees.

\section{Professional Considerations}

According to the literature, one of the reasons that academics, especially doctoral students, postdoctoral researchers, and non-tenure track professors (all precarious workers) may feel discomfort when talking about traumatic or distressing events and emotions, is their lack of job security. Paulson et al. (2009) found that students and faculty may worry about the legitimacy of their trauma during data collection, or the process of disclosing these crises, for fear of endangering their reputation or being labeled as difficult or not able to cope. Dominey-Howes (2015) similarly recognized that his $\mathrm{PhD}$ students and postdoctoral research colleagues likely did not want to speak with him or other faculty members about emotional difficulties, especially if they were trying to find (or keep) a job or succeed within the university. Moreover, the pressure to 
present one's self as "professional" and "self-reliant" or "resilient" impacts how returned students recount their time abroad (often sanitized and romanticized) at pre-departure trainings, furthering the silence on authentic experiences (Pollard 2009).

\section{Institutional Response}

Institutional responses relate to training, resourcing, and communication, while departmental responses can more adequately address methodological and curriculum based concerns. While some institutions have responded to the mental health needs of their students, these interventions often occur at the university level and are not always tailored to the needs of individual disciplines, such as the field of global health (Parizeau et al. 2016). As there is scant literature addressing the mental well-being for post-secondary global health students, current students and faculty have offered suggestions, so that future students may be provided with better support: more rigorous departmental level training, risk assessment in methodological courses, continuous debriefing, regular communication with supervisors, consideration to work with a partner, building in time for breaks from fieldwork, information on available after-care (e.g., university counseling services), budget inclusion for counseling services, therapeutic support for adjusting upon return, open discussions on returning home, validating and normalizing reflective discussions, highlight and support greater self-care, increased and varied social support and networks, supervisorial trainings, researcher safety protocols, standardized global health competencies, designated departmental faculty member focused on student welfare, un-stigmatized leave policy option, and medical leave with benefits (Calgaro 2015; Clark and Grant 2015; Dominey-Howes 2015; Drozdzewski and Dominey-Howes 2015; Huang 2016; Johansson 2015; Matheson et al. 2014; Paulson et al. 2009; Serekoane et al. 2014). An emerging conceptual framework, (Fig. 1) grounded in the academic literature, visually represents global health students' mental health and well-being as related to risks, contributing factors, and academic experience.

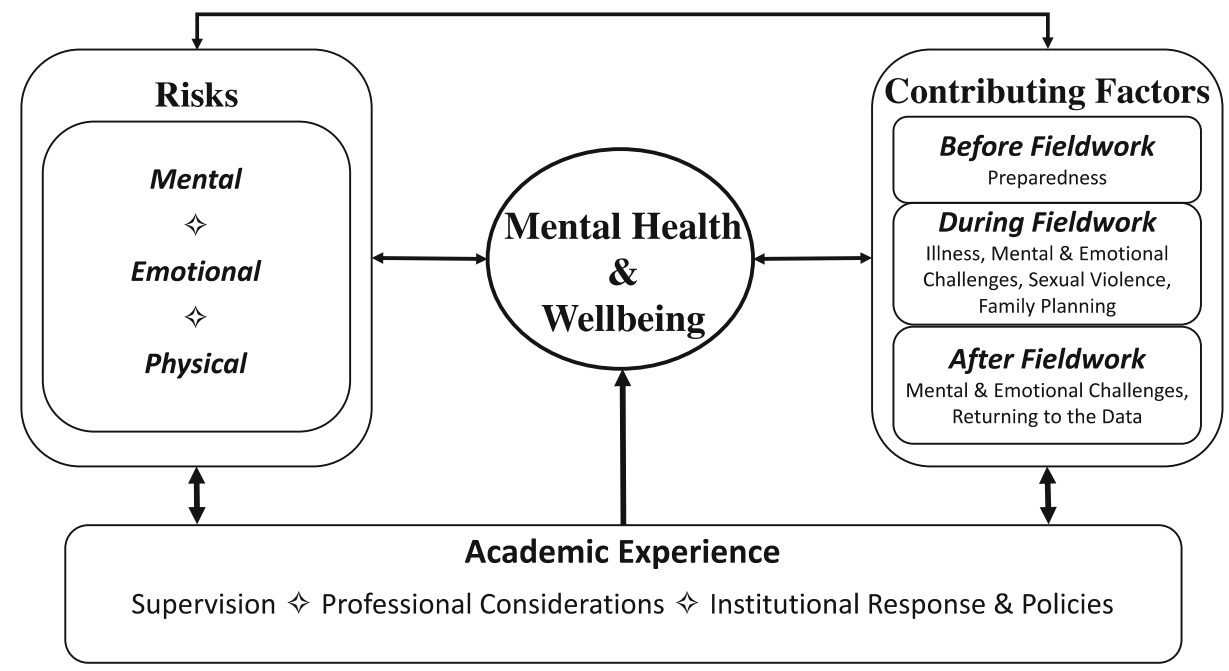

Fig. 1 An emerging conceptual framework grounded in the academic literature. Global health students' mental health and well-being: risks, contributing factors, and academic experience 


\section{Conclusion}

Trauma and distress are part of our human existence and are normal, embodied, personal, and fluid experiences. These events in and of themselves can lead to personal and professional growth through the acquisition of new skills and tools, including accessibility to self-care and better research techniques. Students have reported that fieldwork experiences were transformational and "increased their cultural competency, leadership, coordination, language, teamwork, communication, interpersonal, and program implementation skills" (Donahoe et al. 2017, p. 470). Researchers also recognized the professional benefits they have received due to their experience, such as academic publications, obtaining grants, and personal growth (Clancy et al. 2014; Sohn 2017). According to McLennan et al. (2016), close to two thirds of researchers conducting global health fieldwork had an overall positive experience, even though they reported that it was sometimes unpleasant and distressing.

Furthermore, in Pollard's (2009) research on doctoral students' experience of anthropological fieldwork, she highlighted how several students "described how their time in the field eroded, reshaped or transformed their sense of self"; however, while "some felt that their 'new' self was an improvement, many described the process whereby it had emerged as a traumatic and isolating experience" (p. 5). As Eriksen and Ditrich (2015) note, "we "must take care of ourselves before we can take care of others"' (as cited in Drozdzewski and Dominey-Howes 2015; p. 20). Nevertheless, this is difficult to achieve without appropriate and timely access to varied resources and supports to help foster this continuous process. Trauma to some degree is inevitable. However, there is much room for improvement to lower the likelihood of students experiencing PTSD, secondary-traumatic stress, vicarious trauma, and other serious mental health conditions.

While previous research has focused on the beneficial aspects of experiential learning and fieldwork, there is still much to discover about the physical, mental, and emotional impacts and tolls that global health fieldwork may induce. Issues regarding privilege, racialization, experiences of sexual minorities, and financial concerns and constraints are almost entirely absent from the current literature, and should be addressed in future studies. Additionally, as the clear majority of research in this area concentrates on North American and European populations, future research should focus on the well-being of students and practitioners from low- and middle-income countries. Conclusively, there remains a significant need within the academy to restructure and reform the way in which institutional supports and resources are offered, conducted, and evaluated before, during, and after international student placements.

\section{Compliance with Ethical Standards}

Competing Interests The authors declare that they have no competing interests.

Data Sharing This paper is a critical reflection of the literature. Thus, all data used in support of the paper can be found within articles referenced.

Ethics Approval and Consent The study for which this literature review falls under received ethics approval from the University of Toronto's Institutional Review Board. All data used within this paper reflects available peer reviewed literature. 


\section{References}

Bauer, N., \& Thomas, M. (2009). The dangerous business of aid: A report on the risks to aid workers in the field. Washington, DC: The John Hopkins University.

Begley, L. (2009). The other side of fieldwork: experiences and challenges of conducting research in the border area of Rwanda/eastern Congo. Anthropology Matters, 11(2), 1-11.

Breslau, N., \& Kessler, R. C. (2001). The stressor criterion in DSM-IV posttraumatic stress disorder: an empirical investigation. Biological Psychiatry, 50(9), 699-704.

Brown, T. M., Cueto, M., \& Fee, E. (2006). The World Health Organization and the transition from "international" to "global" public health. American Journal of Public Health, 96(1), 62-72.

Calgaro, E. (2015). If you are vulnerable and you know it raise your hand: experiences from working in posttsunami Thailand. Emotion, Space and Society, 17, 45-54.

Cardozo, B. L., Holtz, T. H., Kaiser, R., Gotway, C. A., Ghitis, F., Toomey, E., \& Salama, P. (2005). The mental health of expatriate and Kosovar Albanian humanitarian aid workers. Disasters, 29(2), 152-170.

Center for Global Health. (n.d.). Women in Global Health Research Initiative. Retrieved from https://www. womenglobalhealth.com/about-us

Clancy, K. B., Nelson, R. G., Rutherford, J. N., \& Hinde, K. (2014). Survey of academic field experiences (SAFE): trainees report harassment and assault. PLoS One, 9(7), e102172.

Clark, I., \& Grant, A. (2015). Sexuality and danger in the field: starting an uncomfortable conversation. Journal of the Anthropological Society of Oxford: Special Issue on Sexual Harassment in the Field, 7(1), 1-14.

Cohen, K., \& Collens, P. (2013). The impact of trauma work on trauma workers: a metasynthesis on vicarious trauma and vicarious posttraumatic growth. Psychological Trauma Theory Research Practice and Policy, 5(6), 570-580.

Congdon, V. (2015). The 'lone female researcher': isolation and safety upon arrival in the field. Journal of the Anthropological Society of Oxford, 7(1), 15-24.

Connolly, K., \& Reilly, R. C. (2007). Emergent issues when researching trauma: a confessional tale. Qualitative Inquiry, 13(4), 522-540.

Connorton, E., Perry, M. J., Hemenway, D., \& Miller, M. (2012). Humanitarian relief workers and trauma-related mental illness. Epidemiologic Reviews, 34(1), 145-155.

Delamont, S. (2009). Familiar screams: a brief comment on "field of screams". Anthropology Matters, 11(2), 12.

Di Ruggiero, E, MacPherson, D, Bajwa, U. (2018) Directions in Global Public Health Graduate Education. In Oxford Bibliographies in Public Health. (Ed.). New York: Oxford University Press. (in press)

Dickson-Swift, V., James, E. L., Kippen, S., \& Liamputtong, P. (2007). Doing sensitive research: what challenges do qualitative researchers face? Qualitative Research, 7(3), 327-353.

Ditzler, T. (2001). Mental health and aid workers: the case for collaborative questioning. Journal of Humanitarian Assistance. Retrieved from https://sites.tufts.edu/jha/archives/1482

Dominey-Howes, D. (2015). Seeing 'the dark passenger'-reflections on the emotional trauma of conducting post-disaster research. Emotion, Space and Society, 17, 55-62.

Donahoe, E. L. H., Rochat, R. W., McFarland, D., \& del Rio, C. (2017). From Albania to Zimbabwe: surveying 10 years of summer field experiences at the Rollins School of public health. Global Health: Science and Practice, 5(3), 468-475.

Drozdzewski, D., \& Dominey-Howes, D. (2015). Research and trauma: understanding the impact of traumatic content and places on the researcher. Emotion, Space and Society, 17, 17-21.

England, K. V. (1994). Getting personal: reflexivity, positionality, and feminist research. The Professional Geographer, 46(1), 80-89.

Eriksen, C., \& Ditrich, T. (2015). The relevance of mindfulness practice for trauma-exposed disaster researchers. Emotion, Space and Society, 17, 63-69.

Grieser, A. (2014). Fieldwork encounters: being foreign and female in Gilgit. Ethnoscripts, 16(1).

Hanson, R., \& Richards, P. (2017). Sexual harassment and the construction of ethnographic knowledge. Sociological Forum, 32(3), 587-609.

Hopwood, N., \& Paulson, J. (2012). Bodies in narratives of doctoral students' learning and experience. Studies in Higher Education, 37(6), 667-681.

Huang, M. (2016). Vulnerable observers: Notes on fieldwork and rape. The Chronicle of Higher Education. Retrieved from https://www.chronicle.com/article/Vulnerable-Observers-Notes-on/238042

Huffman, D. M., \& Rittenmeyer, L. (2012). How professional nurses working in hospital environments experience moral distress: a systematic review. Critical Care Nursing Clinics of North America, 24(1), 91-100.

Inter-Agency Standing Committee. (2006). IASC guidelines on mental health and psychosocial support in emergency settings. Geneva: IASC. 
Jenkins, S. R., \& Baird, S. (2002). Secondary traumatic stress and vicarious trauma: a validational study. Journal of Traumatic Stress, 15(5), 423-432.

Johansson, L. (2015). Dangerous liaisons: risk, positionality and power in women's anthropological fieldwork. Journal of the Anthropological Society of Oxford, 7(1), 55-63.

Kiyimba, N., \& O'Reilly, M. (2015). The risk of secondary traumatic stress in the qualitative transcription process: a research note. Qualitative Research, 92-108.

Klocker, N. (2015). Participatory action research: the distress of (not) making a difference. Emotion, Space and Society, 17, 37-44.

Kloß, S. T. (2016). Sexual(ized) harassment and ethnographic fieldwork: a silenced aspect of social research. Ethnography, 18(3), 396-414.

Koplan, J. P., Bond, T. C., Merson, M. H., Reddy, K. S., Rodriguez, M. H., Sewankambo, N. K., \& Wasserheit, J. N. (2009). Towards a common definition of global health. The Lancet, 373(9679), 1993-1995.

Levecque, K., Anseel, F., De Beuckelaer, A., Van der Heyden, J., \& Gisle, L. (2017). Work organization and mental health problems in PhD students. Research Policy, 46(4), 868-879.

Lowery, K., \& Stokes, M. A. (2005). Role of peer support and emotional expression on posttraumatic stress disorder in student paramedics. Journal of Traumatic Stress, 18(2), 171-179.

Macfarlane, S. B., Jacobs, M., \& Kaaya, E. E. (2008). In the name of global health: trends in academic institutions. Journal of Public Health Policy, 29(4), 383-401.

Matheson, A. I., Pfeiffer, J., Walson, J. L., \& Holmes, K. (2014). Sustainability and growth of university global health programs. Retrieved from The University of Washington Department of Global Health, Center for Strategic and International Studies: http://csis.org/publication/sustainability-and-growth-university-globalhealth-programs.

McKinzie, A. E. (2017). Scared to death: reflections on panic and anxiety in the field. Symbolic Interaction, 40(4), 483-497.

McLennan, J., Evans, L., Cowlishaw, S., Pamment, L., \& Wright, L. (2016). Secondary traumatic stress in postdisaster field research interviewers. Journal of Traumatic Stress.

Mountz, A., Bonds, A., Mansfield, B., Loyd, J., Hyndman, J., Walton-Roberts, M., ... Hamilton, T. (2015). For slow scholarship: a feminist politics of resistance through collective action in the neoliberal university. ACME: An International E-journal for Critical Geographies, 14(4), 1235-1259.

National Council on Disability. (2018). Mental health on college campuses: Investments, accommodations needed to address student needs. Retrieved from Washington, DC: https://ncd.gov/sites/default/files/NCD_ Mental_Health_Report_508_0.pdf.

Parizeau, K., Shillington, L., Hawkins, R., Sultana, F., Mountz, A., Mullings, B., \& Peake, L. (2016). Breaking the silence: a feminist call to action. The Canadian Geographer/Le Géographe canadien, 60(2), 192-204.

Paulson, J., Hopwood, N., McAlpine, L., \& Mills, D. (2009). Untold doctoral experiences: research findings and recommendations from a study into the challenges of doctoral study.

Peake, L. J., \& Mullings, B. (2016). Critical reflections on mental and emotional distress in the academy. ACME: An International Journal for Critical Geographies, 15(2), 253-284.

Pettit, E. (2016). Stigma, stress, and fear: Faculty mental-health services fall short. The Chronicle of Higher Education. Retrieved from https://www.chronicle.com/article/Stigma-StressFear-/237353

Pinto, A. D., \& Upshur, R. E. (2013). An introduction to global health ethics. Abingdon, UK: Routledge.

Pollard, A. (2009). Field of screams: difficulty and ethnographic fieldwork. Anthropology Matters, 11(2), 1-23.

Ross, K. (2015). "No sir, she was not a fool in the field": gendered risks and sexual violence in immersed crosscultural fieldwork. The Professional Geographer, 67(2), 180-186.

Rowley, E. A., Crape, B. L., \& Burnham, G. M. (2007). Violence-related mortality and morbidity of humanitarian workers. American Journal of Disaster Medicine, 3(1), 39-45.

Schwedler, J. (2006). The third gender: Western female researchers in the Middle East. PS: Political Science \& Politics, 39(3), 425-428.

Serekoane, M., Sharp, C., Skinner, D., \& Marais, L. (2014). Untold stories of fieldworkers working amid adverse conditions. Journal of Ethnographic \& Qualitative Research, 8(3), 157-172.

Sharp, G., \& Kremer, E. (2006). The safety dance: confronting harassment, intimidation, and violence in the field. Sociological Methodology, 36(1), 317-327.

Sohn, E. (2017). Health and safety: danger zone. Nature, 541(7636), 247-249.

Surya, M., Jaff, D., Stilwell, B., \& Schubert, J. (2017). The importance of mental well-being for health professionals during complex emergencies: it is time we take it seriously. Global Health: Science and Practice, 5(2), 188-196.

Tucker, F., \& Horton, J. (2018). "The show must go on!” fieldwork, mental health and wellbeing in geography, Earth and Environmental Sciences. Area, 1-10. https://doi.org/10.1111/area.12437.

Welton-Mitchell, C. E. (2013). UNHCR's mental health and psychosocial support for staff. Retrieved from Geneva: UNHCR. 